\title{
OPTIMAL CONTROL OF A TWO TEAMS PREY-PREDATOR INTERACTION MODEL
}

\author{
M. F. ELETTREBY AND SHABAN ALY \\ This paper is dedicated to the memory of Professor Miklos Farkas . \\ Received 11 December, 2013
}

\begin{abstract}
In this paper, a model of two teams of predators interacting with two teams of preys is proposed. The predator teams help each other and so do the prey teams. Therefore, some stability analysis are carried out on the model to detect the stability and instability of the system's equilibrium solutions. Also, the persistence of the solutions is investigated. Finally, the optimal control is applied to the unstable equilibrium solutions. Some numerical simulations are carried out to verify the analytic results.
\end{abstract}

2010 Mathematics Subject Classification: 34C23; 35K57; 92D25; 92D30

Keywords: predator, prey, equilibrium solutions, asymptotic stability, persistence, optimal control

\section{INTRODUCTION}

The work of Malthus [10] and Verhulst [13] is the base to the field of the growth and decline of a population [3,16]. In the nature, species do not exist alone. So, there will be interactions with the other species. When species interact, the population dynamics of each species is affected. There are three main types of interaction [7,11]:

(1) If the growth rate of one population is increased while the growth rate of the other population decreased, then the two populations are in a predator-prey situation.

(2) If the growth rate of each population is decreased affected by the other species, then the two populations are in a competition case.

(3) If the growth rate of each population is enhanced affected by the other species, then it is a mutualism situation.

The work that was proposed independently by Lotka [9] and Volterra [14] opened the gate for studying the predator-prey interaction. Lotka-Volterra model is the simplest model of these interactions. Since their pioneering work, many other notable contributions were made $[12,15]$. 
Many creatures form teams. This has, at least, two main advantages: the first is the improvement in foraging, since looking for food in a team is more efficient than doing it alone. The second advantage is that living in a team reduces predation risk due to early spotting of predators and that existing in a team gives a higher probability that the predator will attack another member of the team. In this paper, a model is given where two teams of predators interact with two teams of preys. The teams of each group (predators or preys) help each other.

A biological realization of our study is represented by herds of zebras and gazelles living side by side and attacked by two types of predators (like lions and tigers). Multi-team game theory has been studied previously in [1,2].

The model is presented in Section 2. The equilibrium points, their local stability and persistence are studied in Section 3. In Section 4, an optimal control to the model is investigated.

\section{THE MODEL}

Consider two teams of preys with densities $x_{1}, x_{2}$ interacting with two teams of predators with densities $y_{1}, y_{2}$, respectively. We assume that, in the absence of the predators, each team of preys grows logistically, $a_{i} x_{i}\left(1-x_{i}\right)$. Each team of the preys will contribute to the growth of the other team, $a_{i} x_{j}, i \neq j$. Also, the two teams of preys help each other against the predator. The effect of the predation is to reduce the prey growth rate by a term proportional to the prey and predator populations, $x_{i}\left(y_{1}+y_{2}\right)$. Finally, in the absence of any prey for sustenance, the predators death rate results in inverse decay, $c_{i} y_{i}$. Using these assumptions, the following model is proposed [6];

$$
\begin{aligned}
& \frac{d x_{1}}{d t}=a_{1}\left[x_{1}\left(1-x_{1}\right)+x_{2}\right]-x_{1}\left(y_{1}+y_{2}\right), \\
& \frac{d x_{2}}{d t}=a_{2}\left[x_{2}\left(1-x_{2}\right)+x_{1}\right]-x_{2}\left(y_{1}+y_{2}\right), \\
& \frac{d y_{1}}{d t}=-c_{1} y_{1}+\left(x_{1}+x_{2}\right)\left(y_{1}+y_{2}\right), \\
& \frac{d y_{2}}{d t}=-c_{2} y_{2}+\left(x_{1}+x_{2}\right)\left(y_{1}+y_{2}\right),
\end{aligned}
$$

where the positive coefficients $a_{1}$ and $a_{2}$ represent the intrinsic growth rates of the preys densities $x_{1}$ and $x_{2}$, respectively. Both the positive parameters $c_{1}$ and $c_{2}$ are the death rates of the predator $y_{1}$ and $y_{2}$, respectively. It is clear that the two preys (predators) help each other e.g. in foraging and (in the case of preys) in early warning against predation. 


\section{THE ANALYSIS OF THE MODEL}

The steady state (equilibrium) solutions are important [4]. The system (2.1) has the following equilibria. The trivial one $E_{1}=(0,0,0,0)$, the boundary one $E_{2}=$ $(2,2,0,0)$ and the coexistence (interior) $E_{3}=\left(x_{1}, x_{2}, y_{1}, y_{2}\right)$. The components of the interior solutions are given by the relations;

$$
y_{2}=\frac{c_{1}}{c_{2}} y_{1}, y_{1}=\frac{a_{1} c}{x_{1} c_{1}}\left(c-x_{1}^{2}\right), x_{2}=\left(c-x_{1}\right),
$$

and

$$
x_{1}^{3}-c\left(1+A_{2}\right) x_{1}^{2}-c\left(1-c A_{2}\right) x_{1}+c^{2} A_{1}=0
$$

where

$$
A_{1}=\frac{a_{1}}{a_{1}+a_{2}}, \quad A_{2}=\frac{a_{2}}{a_{1}+a_{2}} \text { and } c=\frac{c_{1} c_{2}}{c_{1}+c_{2}} .
$$

Proposition 1. The system (2.1) has at least one and at most two interior equilibrium solutions [6].

Proposition 2. Local stability analysis [4] shows that the equilibrium state $E_{1}=$ $(0,0,0,0)$ is unstable, while $E_{2}=(2,2,0,0)$ is stable under the conditions $c_{1}+c_{2}>$ 8 , and $4\left(c_{1}+c_{2}\right)<c_{1} c_{2}$.

Proof. The Jacobian matrix of the system (2.1) is given by $J=$

$$
\left[\begin{array}{llll}
a_{1}\left(1-2 x_{1}\right)-\left(y_{1}+y_{2}\right) & a_{1} & -x_{1} & -x_{1} \\
a_{2} & a_{2}\left(1-2 x_{2}\right)-\left(y_{1}+y_{2}\right) & -x_{2} & -x_{2} \\
\left(y_{1}+y_{2}\right) & \left(y_{1}+y_{2}\right) & \left(x_{1}+x_{2}\right)-c_{1} & \left(x_{1}+x_{2}\right) \\
\left(y_{1}+y_{2}\right) & \left(y_{1}+y_{2}\right) & \left(x_{1}+x_{2}\right) & \left(x_{1}+x_{2}\right)-c_{2}
\end{array}\right] .
$$

At the equilibrium point $E_{1}=(0,0,0,0)$, the above Jacobian matrix becomes;

$$
J=\left[\begin{array}{llll}
a_{1} & a_{1} & 0 & 0 \\
a_{2} & a_{2} & 0 & 0 \\
0 & 0 & -c_{1} & 0 \\
0 & 0 & 0 & -c_{2}
\end{array}\right],
$$

whose eigenvalues are $\lambda=0,-c_{1},-c_{1}, a_{1}+a_{2}$, which has one positive eigenvalue, $a_{1}+a_{2}>0$. So, it is unstable equilibrium point.

Similarly, at the equilibrium point $E_{2}=(2,2,0,0)$, The Jacobian matrix becomes;

$$
J=\left[\begin{array}{llll}
-3 a_{1} & a_{1} & -2 & -2 \\
a_{2} & -3 a_{2} & -2 & -2 \\
0 & 0 & 4-c_{1} & 4 \\
0 & 0 & 4 & 4-c_{2}
\end{array}\right]
$$

whose eigenvalues satisfy the characteristic equations;

$$
\lambda^{2}+3\left(a_{1}+a_{2}\right) \lambda+8 a_{1} a_{2}=0,
$$


and

$$
\lambda^{2}-\left(8-c_{1}-c_{2}\right) \lambda+c_{1} c_{2}-4\left(c_{1}+c_{2}\right)=0 .
$$

The first equation has two negative eigenvalues since $3\left(a_{1}+a_{2}\right)>0,8 a_{1} a_{2}>0$ and the two other eigenvalues come from the second equation which are negative under the conditions

$$
c_{1}+c_{2}>8, \quad 4\left(c_{1}+c_{2}\right)<c_{1} c_{2}
$$

Definition 1. A system is persistent if there exists a compact region $V$ subset of the interior of the state space such that all solutions with positive initial conditions are attracted to $V[8]$.

Proposition 3. The system (2.1) is persistent if at least one of the above conditions is not satisfied [6].

The characteristic equation of the eigenvalue of the internal equilibrium point $E_{3}$ is given by, $\lambda^{4}+D_{1} \lambda^{3}+D_{2} \lambda^{2}+D_{3} \lambda+D_{4}=0$, where

$$
\begin{aligned}
D_{1}= & \left(-2 a_{2}\right) x_{1}+\left(c_{1}+c_{2}-a_{1}-a_{2}+2 a_{2} c-2 c\right)+\left(2 a_{1} c\right) x_{1}^{-1} \\
D_{2}= & \left(2 a_{1}-a_{1}^{2}-2 a_{1} a_{2}\right) x_{1}^{2} \\
& +\left(4 a_{2} c+2 a_{1} a_{2} c+a_{1} a_{2}+a_{1}^{2}-2 a_{1} c-2 a_{2} c_{1}-2 a_{2} c_{2}\right) x_{1} \\
& -\left(4 a_{1} a_{2} c+4 a_{2} c^{2}+a_{1} c_{1}+a_{1} c_{2}+a_{2} c_{1}+a_{2} c_{2}-2 a_{2} c c_{1}-2 a_{2} c c_{2}-2 a_{2} c\right) \\
& +\left(a_{1} c\right)\left(2 c_{1}+2 c_{2}+2 a_{2} c-a_{1}-a_{2}-2 c\right) x_{1}^{-1}+\left(a_{1}^{2} c^{2}\right) x_{1}^{-2} \\
D_{3}= & \left(2 a_{1}^{2}\right) x_{1}^{3}+a_{1}\left(4 a_{2} c+2 c_{1}-2 a_{2} c_{1}-2 a_{2} c_{2}-a_{1} c_{1}-a_{1} c_{2}\right) x_{1}^{2} \\
& -a_{1}\left(c\left(c_{1}+c_{2}\right)+\left(a_{1}+a_{2}+2 a_{2} c\right)\left(2 c-c_{1}-c_{2}\right)\right) x_{1}+\left(2 a_{1} c\right) \\
& \left(2 a_{2}\left(2 c-c_{1}-c_{2}\right)-c_{1}\right)+\left(a_{1} c\right)\left(\left(2 c-c_{1}-c_{2}\right)\left(a_{1}+a_{2}-2 a_{2} c\right)\right. \\
& \left.+c\left(c_{1}+c_{2}\right)-2 a_{1} c\right) x_{1}^{-1}+\left(a_{1}^{2} c c_{1} c_{2}\right) x_{1}^{-2} \\
D_{4}= & \left(2 a_{1}\right)\left(a_{1} c_{2}-a_{2} c_{1}+a_{2} c_{2}\right) x_{1}^{3}-\left(a_{1} c\right)\left(a_{1}\left(c_{1}+c_{2}\right)+2 a_{2}\left(c_{2}-c_{1}\right)\right) x_{1}^{2} \\
& +\left(2 a_{1} c\right)\left(a_{1}+a_{2}\right)\left(c_{1}-c_{2}\right) x_{1}+\left(2 a_{1} a_{2} c^{2}\right)\left(c_{2}-c_{1}\right)-\left(2 a_{1}^{2} c^{2} c_{1}\right) x_{1}^{-1} \\
& +\left(a_{1}^{2} c^{3}\right)\left(c_{1}+c_{2}\right) x_{1}^{-2}
\end{aligned}
$$

The Routh-Hurwitz criteria [4] shows that the interior point $E_{3}\left(x_{1}, x_{2}, y_{1}, y_{2}\right)$ is locally asymptotically stable if the determinants of all Hurwitz matrices are positive where the Hurwitz matrices are given by;

$$
\begin{aligned}
& H_{1}=\left(D_{1}\right) \quad H_{2}=\left(\begin{array}{cc}
D_{1} & 1 \\
D_{3} & D_{2}
\end{array}\right) \\
& H_{3}=\left(\begin{array}{ccc}
D_{1} & 1 & 0 \\
D_{3} & D_{2} & D_{1} \\
0 & D_{4} & D_{3}
\end{array}\right) \quad H_{4}=\left(\begin{array}{cccc}
D_{1} & 1 & 0 & 0 \\
D_{3} & D_{2} & D_{1} & 1 \\
0 & D_{4} & D_{3} & D_{2} \\
0 & 0 & 0 & D_{4}
\end{array}\right)
\end{aligned}
$$


Using different values of the constants $a_{1}, a_{2}, c_{1}$ and $c_{2}$ to find the roots of equation (3.1), one gets that one of them is negative and one of the other two positive roots is greater than $c$ i.e $x_{2}$ will be negative. So, we have only one interior equilibrium point.

Let the initial values of the preys and the predators are, respectively, $x_{1}(0)=0.1$, $x_{2}(0)=0.4, y_{1}(0)=0.3, y_{2}(0)=0.7$, and the values of the parameters of the model are $a_{1}=1.2, a_{2}=1.7, c_{1}=0.3$ and $c_{2}=0.5$. Substituting by these values in equations $(3.2,3.1)$, we get the interior equilibrium point $E_{3}=(0.079,0.1085,1.7208$, 1.0325), which is asymptotically stable since there are two negative real eigenvalues $\lambda=-3.019684381,-0.4022762114$ and another two conjugate complex eigenvalues with negative real parts $\lambda=-0.08409198332 \pm 1.009538530 I$. We simulate the model (2.1) for the mentioned initial values of the densities, we found that the system converges to the asymptotically stable point $E_{3}$ as shown in Fig. 1 and Fig. 2.
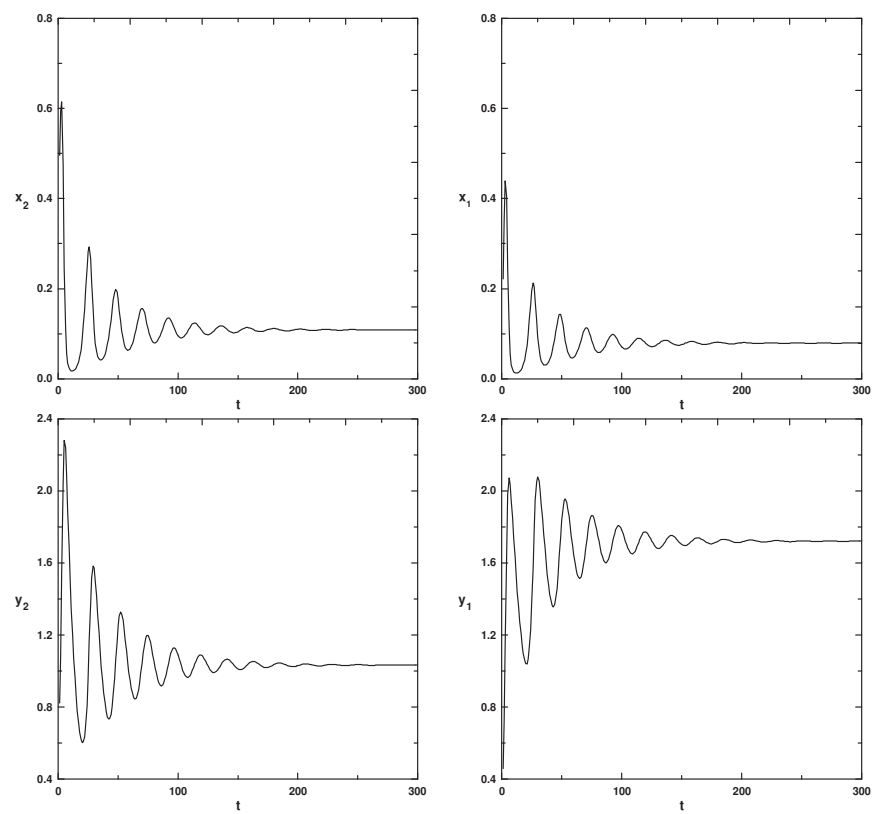

FIGURE 1. The densities of the preys and predators with time at the parameter values $a_{1}=1.2, a_{2}=1.7, c_{1}=0.3, c_{2}=0.5$ and the initial densities $x_{1}(0)=0.1, x_{2}(0)=0.4, y_{1}(0)=0.3, y_{2}(0)=0.7$.

\section{OPTIMAL CONTROL PROBLEM}

In some cases the presence of the predators is harmful. So, we should keep the situation in which the predator does not exist. Such as the case when the predators are viruses or tumors. Also, there are some animals that present in few numbers, like 

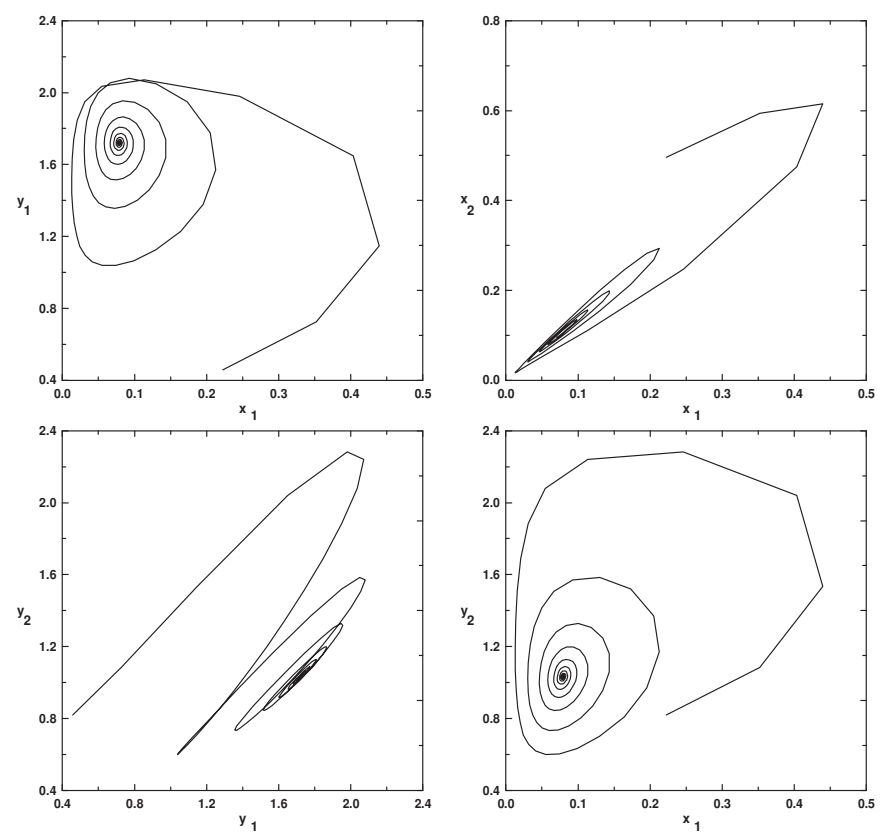

FIGURE 2. The system converges to the stable point $E_{3}$ for the parameter values $a_{1}=1.2, a_{2}=1.7, c_{1}=0.3, c_{2}=0.5$ and the initial densities $x_{1}(0)=0.1, x_{2}(0)=0.4, y_{1}(0)=0.3, y_{2}(0)=0.7$.

Panda, we have to keep them from extinction or predation. In cases like these, it is required to make these equilibrium solutions stable.

We saw that the first equilibrium solution $E_{1}$ is unstable and the second equilibrium solution $E_{2}$ is unstable if one of the conditions $c_{1}+c_{2}>8$ or $4\left(c_{1}+c_{2}\right)<c_{1} c_{2}$ is not satisfied. Then, we will use the optimal control technique to stabilize the second solution $E_{2}$ in this section.

Here, we will use the same feedback control approach which is presented in [5] for a different model. Consider the above system in the following form;

$$
\begin{aligned}
& \frac{d x_{1}}{d t}=a_{1}\left[x_{1}\left(1-x_{1}\right)+x_{2}\right]-x_{1}\left(y_{1}+y_{2}\right)+u_{1}, \\
& \frac{d x_{2}}{d t}=a_{2}\left[x_{2}\left(1-x_{2}\right)+x_{1}\right]-x_{2}\left(y_{1}+y_{2}\right)+u_{2}, \\
& \frac{d y_{1}}{d t}=-c_{1} y_{1}+\left(x_{1}+x_{2}\right)\left(y_{1}+y_{2}\right)+u_{3}, \\
& \frac{d y_{2}}{d t}=-c_{2} y_{2}+\left(x_{1}+x_{2}\right)\left(y_{1}+y_{2}\right)+u_{4},
\end{aligned}
$$


where $u_{i},(i=1,2,3,4)$, are the control inputs. Some performance measure is used to determine these inputs from the conditions of asymptotic stability of the system (2.1) about its unstable equilibrium states.

Let

$$
z_{1}=x_{1}-\bar{x}_{1}, z_{2}=x_{2}-\bar{x}_{2}, w_{1}=y_{1}-\bar{y}_{1}, w_{2}=y_{2}-\bar{y}_{2} \text {, }
$$

where $z_{1}, z_{2}, w_{1}, w_{2}$ represent perturbed states about its unstable equilibrium states and $\left(\bar{x}_{1}, \bar{x}_{2}, \bar{y}_{1}, \bar{y}_{2}\right)$ denote to the coordinates of the unstable equilibrium states of system (2.1). Substituting by (4.2) into the system (4.1) taking into account the identities that satisfied by the equilibrium states, we get the following system,

$$
\begin{aligned}
& \dot{z}_{1}=-\left(2 a_{1} \bar{x}_{1}+\bar{y}_{1}+\bar{y}_{2}\right) z_{1}+a_{1}\left(z_{1}+z_{2}-z_{1}^{2}\right)-\left(\bar{x}_{1}+z_{1}\right)\left(w_{1}+w_{2}\right)+u_{1}, \\
& \dot{z}_{2}=-\left(2 a_{2} \bar{x}_{2}+\bar{y}_{1}+\bar{y}_{2}\right) z_{2}+a_{2}\left(z_{1}+z_{2}-z_{2}^{2}\right)-\left(\bar{x}_{2}+z_{2}\right)\left(w_{1}+w_{2}\right)+u_{2}, \\
& \dot{w}_{1}=-c_{1} w_{1}+\left(z_{1}+z_{2}\right)\left(w_{1}+w_{2}+\bar{y}_{1}+\bar{y}_{2}\right)+\left(\bar{x}_{1}+\bar{x}_{2}\right)\left(w_{1}+w_{2}\right)+u_{3}, \\
& \dot{w}_{2}=-c_{2} w_{2}+\left(z_{1}+z_{2}\right)\left(w_{1}+w_{2}+\bar{y}_{1}+\bar{y}_{2}\right)+\left(\bar{x}_{1}+\bar{x}_{2}\right)\left(w_{1}+w_{2}\right)+u_{4} .
\end{aligned}
$$

The system (4.3) admits the trivial solution,

$$
z_{1}=z_{2}=w_{1}=w_{2}=0, u_{1}=u_{2}=u_{3}=u_{4}=0 .
$$

This solution (from equation (4.2)) represents the equilibrium states of the system (2.1).

Theorem 1. Using the optimal nonlinear control inputs;

$$
\begin{aligned}
& u_{1}^{*}=-a_{1}\left(z_{1}+z_{2}-z_{1}^{2}\right)+\left(\bar{x}_{1}+z_{1}\right)\left(w_{1}+w_{2}\right), \\
& u_{2}^{*}=-a_{2}\left(z_{1}+z_{2}-z_{2}^{2}\right)+\left(\bar{x}_{2}+z_{2}\right)\left(w_{1}+w_{2}\right), \\
& u_{3}^{*}=-\left(z_{1}+z_{2}\right)\left(w_{1}+w_{2}+\bar{y}_{1}+\bar{y}_{2}\right)-\left(\bar{x}_{1}+\bar{x}_{2}\right)\left(w_{1}+w_{2}\right), \\
& u_{4}^{*}=-\left(z_{1}+z_{2}\right)\left(w_{1}+w_{2}+\bar{y}_{1}+\bar{y}_{2}\right)-\left(\bar{x}_{1}+\bar{x}_{2}\right)\left(w_{1}+w_{2}\right),
\end{aligned}
$$

the unstable equilibrium states of the system (2.1) will be optimally asymptotically stable with respect to the performance measure;

$$
\begin{aligned}
I= & \int_{0}^{\infty} \Omega d t \\
= & \int_{0}^{\infty}\left\{\left[u_{1}+a_{1}\left(z_{1}+z_{2}-z_{1}^{2}\right)-\left(\bar{x}_{1}+z_{1}\right)\left(w_{1}+w_{2}\right)\right]^{2}\right. \\
& +\left[u_{2}+a_{2}\left(z_{1}+z_{2}-z_{2}^{2}\right)-\left(\bar{x}_{2}+z_{2}\right)\left(w_{1}+w_{2}\right)\right]^{2} \\
& +\left[u_{3}+\left(z_{1}+z_{2}\right)\left(w_{1}+w_{2}+\bar{y}_{1}+\bar{y}_{2}\right)+\left(\bar{x}_{1}+\bar{x}_{2}\right)\left(w_{1}+w_{2}\right)\right]^{2} \\
& +\left[u_{4}+\left(z_{1}+z_{2}\right)\left(w_{1}+w_{2}+\bar{y}_{1}+\bar{y}_{2}\right)+\left(\bar{x}_{1}+\bar{x}_{2}\right)\left(w_{1}+w_{2}\right)\right]^{2} \\
& \left.+\left(2 a_{1} \bar{x}_{1}+\bar{y}_{1}+\bar{y}_{2}\right) z_{1}^{2}+\left(2 a_{2} \bar{x}_{2}+\bar{y}_{1}+\bar{y}_{2}\right) z_{2}^{2}+c_{1} w_{1}^{2}+c_{2} w_{2}^{2}\right\} d t
\end{aligned}
$$


Proof. We will use Bellman dynamic programming technique to obtain the optimal control inputs and Liapunov asymptotic stability theorem to prove our theorem.

Suppose that we have the nonlinear system (4.3) with the four perturbed state variables $z_{1}, z_{2}, w_{1}, w_{2}$ and the four control inputs $u_{i},(i=1,2,3,4)$ with $\dot{z}_{i}, \dot{w}_{i}$, are definite functions of $z_{i}, w_{i}, i=1,2$, and $u_{j}, j=1,2,3,4$. If we consider the minimization of an integral of the form (4.6) we can write the condition for minimization of the integral of the form;

$$
\begin{aligned}
\min _{\vec{u}} & \left\{\frac{\partial V}{\partial z_{1}} \dot{z}_{1}+\frac{\partial V}{\partial z_{2}} \dot{z}_{2}+\frac{\partial V}{\partial w_{1}} \dot{w}_{1}+\frac{\partial V}{\partial w_{2}} \dot{w}_{2}\right. \\
& +\left[u_{1}+a_{1}\left(z_{1}+z_{2}-z_{1}^{2}\right)-\left(\bar{x}_{1}+z_{1}\right)\left(w_{1}+w_{2}\right)\right]^{2} \\
& +\left[u_{2}+a_{2}\left(z_{1}+z_{2}-z_{2}^{2}\right)-\left(\bar{x}_{2}+z_{2}\right)\left(w_{1}+w_{2}\right)\right]^{2} \\
& +\left[u_{3}+\left(z_{1}+z_{2}\right)\left(w_{1}+w_{2}+\bar{y}_{1}+\bar{y}_{2}\right)+\left(\bar{x}_{1}+\bar{x}_{2}\right)\left(w_{1}+w_{2}\right)\right]^{2} \\
& +\left[u_{4}+\left(z_{1}+z_{2}\right)\left(w_{1}+w_{2}+\bar{y}_{1}+\bar{y}_{2}\right)+\left(\bar{x}_{1}+\bar{x}_{2}\right)\left(w_{1}+w_{2}\right)\right]^{2} \\
& \left.+\left(2 a_{1} \bar{x}_{1}+\bar{y}_{1}+\bar{y}_{2}\right) z_{1}^{2}+\left(2 a_{2} \bar{x}_{2}+\bar{y}_{1}+\bar{y}_{2}\right) z_{2}^{2}+c_{1} w_{1}^{2}+c_{2} w_{2}^{2}\right\}=0 .
\end{aligned}
$$

Now, the function $V$ is a function of $z_{i}, w_{i}, i=1,2$, and it represents the minimum value of the integral performance measure (4.6) achievable by starting from time $t$ and state $\left(z_{1}, z_{2}, w_{1}, w_{2}\right)$. Let us assume that $V$ is the solution of the (4.7) takes the form,

$$
V=z_{1}^{2}+z_{2}^{2}+w_{1}^{2}+w_{2}^{2}
$$

which is a positive definite function of the variables $\left(z_{1}, z_{2}, w_{1}, w_{2}\right)$ and so will be used to prove the asymptotic stability of the solution (4.4).

Substituting by (4.3) and (4.8) in (4.7) and differentiating the resulting expression with respect to $u_{i}$ and setting it to zero, we obtain the optimal control inputs $u_{i}^{*}$ as given by (4.5).

Differentiating the function $V$ along the trajectory of the system (4.3) taking into account the control inputs (4.5) we get;

$$
\begin{aligned}
\dot{V} & =2 z_{1} \dot{z}_{1}+2 z_{2} \dot{z}_{2}+2 w_{1} \dot{w}_{1}++2 w_{1} \dot{w}_{1} \\
& =-2\left\{\left(2 a_{1} \bar{x}_{1}+\bar{y}_{1}+\bar{y}_{2}\right) z_{1}^{2}+\left(2 a_{2} \bar{x}_{2}+\bar{y}_{1}+\bar{y}_{2}\right) z_{2}^{2}+c_{1} w_{1}^{2}+c_{2} w_{2}^{2}\right\} \\
& =-2 \Omega^{*}
\end{aligned}
$$

where $\Omega^{*}$ is the value of the objective function $\Omega$ at the optimal control inputs.

Obviously, the function $\dot{V}$ is a negative definite function of $\left(z_{1}, z_{2}, w_{1}, w_{2}\right)$ only for positive equilibrium states $\bar{x}_{i}, \bar{y}_{i}>0,(i=1,2)$ and so the positive equilibrium states (4.4) are asymptotically stable in the Liapunov sense if the conditions of stability are satisfied. 
Some numerical analysis are achieved to show the stabilization behavior of the system (2.1) at the second unstable equilibrium solution $E_{2}$. This numerical analysis supports the above analytical analysis. The model parameters are fixed as follows: $a_{1}=0.2, a_{2}=0.7, c_{1}=0.3, c_{2}=0.5$. Note that the conditions of stability of $E_{2}$ are not satisfied. The initial densities of preys are $z_{1}=0.1$ and $z_{2}=0.4$ while the initial densities of predators are $w_{1}=0.3$ and $w_{2}=0.7$. A lot of simulations for different values of the parameters and the initial values of the densities are done. Fig. 3 shows that the preys and predator densities perturbations decay to zero. In Fig. 4, the components of the control inputs tend to zero. So, we conclude that the unstable equilibrium solution $E_{2}$ of system (2.1) can be stabilized by using nonlinear control parameters (4.1)
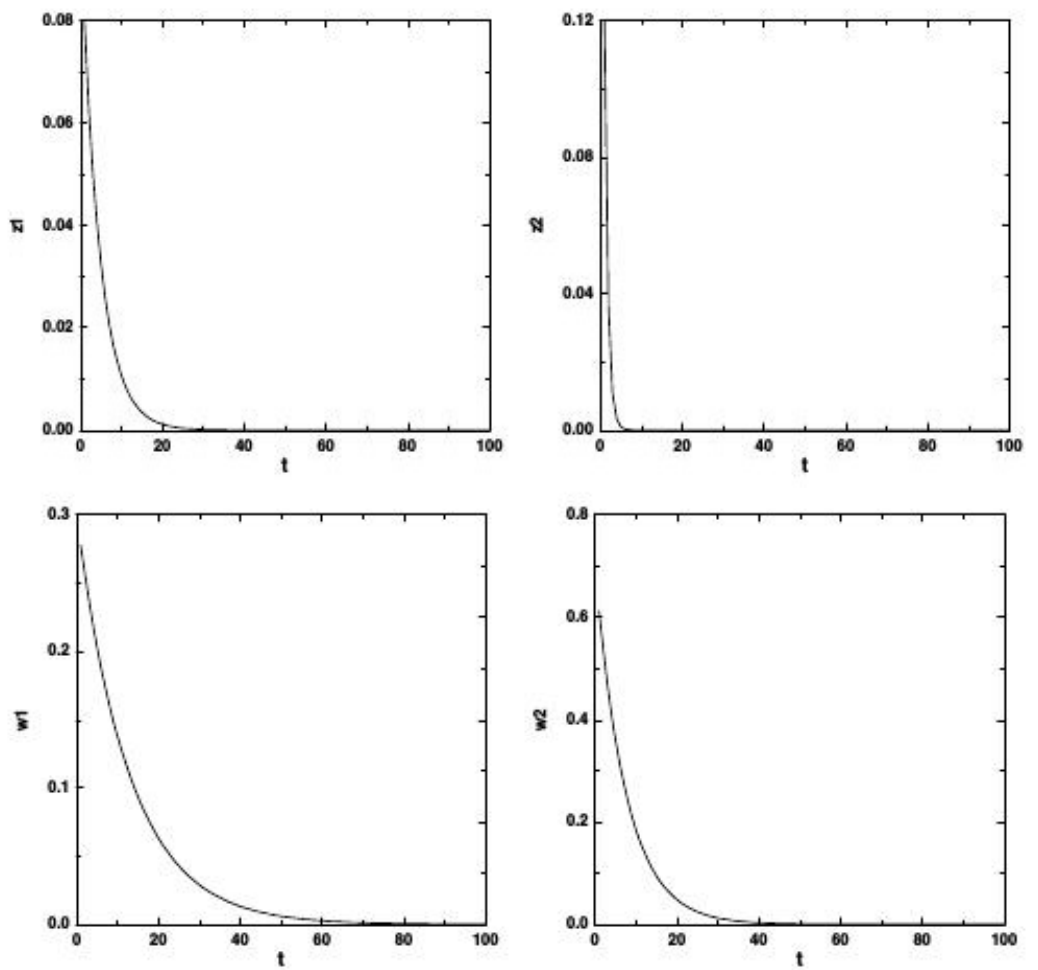

FIGURE 3. The densities perturbations of the preys and predators with time converges to zero at the parameter values $a_{1}=0.2, a_{2}=$ $0.7, c_{1}=0.3, c_{2}=0.5$ and the initial densities $z_{1}(0)=0.1, z_{2}(0)=$ $0.4, w_{1}(0)=0.3, w_{2}(0)=0.7$. 

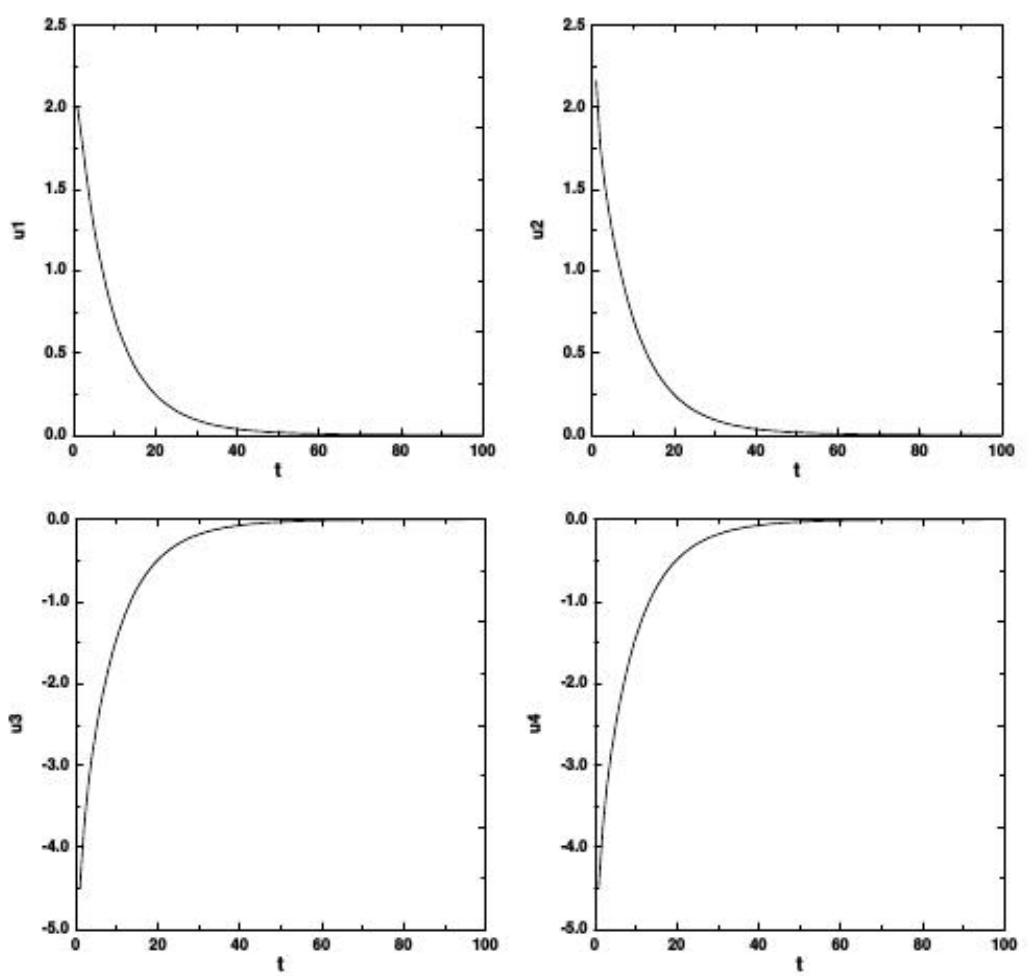

FIGURE 4. The components of the control inputs converges to the stable point state $(0,0,0,0)$ at the parameter values $a_{1}=0.2, a_{2}=$ $0.7, c_{1}=0.3, c_{2}=0.5$ and the initial densities $z_{1}(0)=0.1, z_{2}(0)=$ $0.4, w_{1}(0)=0.3, w_{2}(0)=0.7$.

\section{CONCLUSION}

Many animals live in groups. Different groups share one habitat hence these groups may cooperate, compete with each other or form predator-prey system. In this work we present a model for the interaction between predator-prey teams. Equilibrium solutions are derived, their local stability and persistence. An optimal control is used to stabilized the unstable equilibrium solution. The boundary unstable equilibrium solution $E_{2}$ is stabilized using nonlinear control inputs.

\section{ACKNOWLEDGMENTS}

The authors would like to thank the editor as well as the anonymous referees very much for their invaluable and comprehensive comments which helped in improving the paper. We are very grateful to all members of Applied Mathematics Group 
(Department of Mathematics, Faculty of Science, King Khalid University) for useful discussions on the topics investigated in this paper.

\section{REFERENCES}

[1] E. Ahamed and A. S. Hegazi, "On dynamical multi-team and signaling games," Applied Mathematics and Computation, vol. 172, pp. 524-530, 2006, doi: 10.1016/j.amc.2005.02.030.

[2] E. Ahmed, A. S. Hegazi, M. F. Elettreby, and S. S. Askar, "On multi-team games," Physica A, vol. 369, pp. 809-816, 2006, doi: 10.1016/j.physa.2006.02.011.

[3] J. Cai and D. Mei, "Influence of strong noise on the decline and propagation of population in the delayed malthus-verhulst model," Physica A, vol. 389, pp. 4715-4724, 2010.

[4] L. Edelstein-Keshet, Mathematical Models in Biology. SIAM, 2005.

[5] A. El-Gohary, "Chaos and optimal control of self-remission and tumor system steady states," Chaos, Solitons \& Fractals, vol. 37, pp. 1305-1316, 2008, doi: 10.1016/j.chaos.2006.10.060.

[6] M. F. Elettreby, S. H. Saker, and E. Ahmed, “On multi-team predator-prey models," ictp, pp. 5-23, 2006.

[7] M. Farkas, Mathematical Models in Biology. San Diego, CA: Academic Press, 2001.

[8] J. Hofbauer and K. Sigmund, Evolutionary games and population dynamics. Cambridge: Cambridge University Press, 1998.

[9] A. J. Lotka, Elements of Physical Biology. Williams and Wilkins: Baltimore, 1925.

[10] T. R. Malthus, An Essay on The Principle of Population. L London: J Johnson, 1798.

[11] J. D. Murray, Mathematical Biology I: An Introduction. S Springer 3rd edition, 2007.

[12] G. Seo and D. L. DeAngelis, "A predator-prey model with a holling type i: Functional response including a predator mutual interference," Journal of Nonlinear Science, vol. 21, pp. 811-833, 2011, doi: 10.1007/s00332-011-9101-6.

[13] P. F. Verhulst, "Notice sur la loi que la population suit dans son accroissement," Correspondance Mathematique et Physique, vol. 10, pp. 113-121, 1838.

[14] V. Volterra, Variations and Fluctuations of a Number of Individuals in Animal Species Living Together. Newyork: McgrawHill, 1931.

[15] Q. Zhang, C. Liu, and X. Zhang, "Complex dynamical behavior in bio-economic prey-predator models with competition for prey," Lecture Notes in Control and Information Sciences, vol. 421, pp. 185-201, 2012, doi: 10.1007/978-1-4471-2303-3'13.

[16] M. Zhou and Z. Liu, "Hopf bifurcations in a ricardo-malthus model," Applied Mathematics and Computation, vol. 217, pp. 2425-2432, 2010, doi: 10.1016/j.amc.2010.07.043.

Authors' addresses

\section{F. Elettreby}

Current address: King Khalid University, Faculty of Science, Department of Mathematics, Applied Mathematics Group, Abha 9004, Saudi Arabia

Mansoura University, Faculty of Science, Department of Mathematics, Mansoura 35516, Egypt E-mail address: mohfathy@mans.edu.eg

\section{Shaban Aly}

Current address: King Khalid University, Faculty of Science, Department of Mathematics, Applied Mathematics Group, Abha 9004, Saudi Arabia

Al-Azhar University, Faculty of Science, Mathematics Department, Assiut 71511, Egypt

E-mail address: shhaly70@yahoo.com 